\title{
Eficiencia terminal según modalidad de titulación de pregrado de estomatología en la Universidad Peruana Cayetano Heredia, 1975-2018
}

Terminal efficiency according to undergraduate degree of stomatology at the Universidad Peruana Cayetano Heredia, 1975-2018

Angela Girano-Arévalo ${ }^{1, a}$, Sally Vásquez-Salas ${ }^{1, a}$, Roberto Antonio León-Manco ${ }^{1, b}$, Jorge A. Beltrán ${ }^{1, c}$

RESUMEN

La obtención del título profesional es la última etapa de la formación profesional, sin embargo, cada modalidad tiene sus propias características, y por ello, su propia eficiencia terminal. Objetivo: Determinar la eficiencia terminal según modalidad de titulación de los egresados de pregrado de la Facultad de Estomatología de la Universidad Peruana Cayetano Heredia, Lima-Perú, desde el año 1975 al 2018. Material y Métodos: La investigación fue retrospectiva basada en los registros de los egresados de pregrado según el repositorio institucional de la universidad. Las variables fueron modalidad de titulación, eficiencia terminal y década de egreso. Para el análisis estadístico se usó el programa estadístico STATA 16.1. Resultados: El trabajo de investigación presentó la menor eficiencia terminal con promedio de 1,01 años $(\mathrm{DE}=0,10)$ y con frecuencia de $98,97 \%(\mathrm{n}=96)$ Hasta 1 año, siendo estadísticamente significativos $(\mathrm{p}<0,05)$. Conclusiones: El trabajo de investigación presentó mejor eficiencia terminal en los egresados de pregrado de la Facultad de Estomatología de la Universidad Peruana Cayetano Heredia, Lima-Perú, desde el año 1975 al 2018.

PALABRAS CLAVE: Tesis académica, investigación, estudiantes, educación profesional, salud bucal. 


\section{SUMMARY}

Obtaining the professional title is the last stage of professional training, however, each modality has its own characteristics, and therefore, its own terminal efficiency. Objective: Determine the terminal efficiency according to the degree modality of undergraduate graduates of the Faculty of Stomatology of the Universidad Peruana Cayetano Heredia, Lima-Peru, from 1975 to 2018. Material and Methods: The research was retrospective based on records of undergraduate graduates according to the institutional repository of the university. The variables were degree modality, terminal efficiency and graduation decade. For the statistical analysis, the statistical program STATA 16.1 was used. Results: The research work presented the lowest terminal efficiency with an average of 1.01 years $(\mathrm{SD}=0.10)$ and with a frequency of $98.97 \%(\mathrm{n}=96) \mathrm{Up}$ to 1 year, being statistically significant $(\mathrm{p}<0.05)$. Conclusions: The research work presented better terminal efficiency in undergraduate graduates of the Faculty of Stomatology of the Universidad Peruana Cayetano Heredia, Lima-Peru, from 1975 to 2018 .

KEYWORDS: Academic Dissertation, research, students, professional education, oral health.

\section{INTRODUCCIÓN}

La obtención de grados y títulos en las universidades es la última etapa en la formación profesional (1). En el Perú, la denominada nueva Ley Universitaria $\mathrm{N}^{\circ} 30220$ (NLU) (2), que reemplaza la antigua Ley Universitaria $\mathrm{N}^{\circ} 23733$ (ALU) (3), modifica la modalidad de obtención de grados y títulos de pregrado, indica que tanto para el grado de bachiller (GB) y título profesional (TP) es necesario desarrollar investigaciones, salvo que las facultades se encuentren acreditadas nacionalmente y puedan proponer su propia modalidad de titulación (MT); esta ley es vigente desde los ingresantes del año 2016. En la actualidad, los profesionales presentan dificultades para su inserción laboral, exigiendo que sean mucho más competentes con preparación formal de pre y posgrado; por ello, es indispensable obtener el TP en el menor tiempo posible; es decir, una adecuada eficiencia terminal (ET), que se define como el tiempo transcurrido desde el egreso universitario hasta la obtención del TP (4).

En el caso peruano, aún no se tienen egresados enmarcados en la NLU, por lo cual las tesis, trabajos de suficiencia profesional, trabajos de investigación y otros son necesarios para la obtención del TP, porque el GB sigue siendo automático (2). En este contexto, las investigaciones son la materialización de la generación de conocimiento, se ha visto que esta producción ha aumentado notoriamente; sin embargo, no es precisamente en los países occidentales, por el contrario, las crecientes olas provienen de Asia (5). Las universidades tienen la responsabilidad de aportar conocimientos no solo a sus alumnos, sino también a la sociedad; es necesario entender que, para poder progresar como país, es importante reflejar el desarrollo científico, tecnológico e innovador en todas las áreas que sean posibles, por ello, la formación profesional incluye competencias de investigación (6).

Existen diversos factores que incluyen en la culminación de una investigación desde, económicos, sociales, culturales y de gestión del propio proceso; esto debido a que muchas veces las instituciones contemplan procesos administrativos no comprensibles para los estudiantes y asesores, así como, formatos de investigaciones no acordes con la modernidad que ocasionan retrasos o cancelación de los trabajos (7). A pesar de ello, en el Perú, el proceso de licenciamiento de universidades ha exigido que las instituciones mejoren sus procesos de gestión en investigación, siendo uno de ellos, la modalidad de obtención de grados y títulos con la finalidad de contar con más egresados en el menor tiempo posible, es decir, adecuada eficiencia terminal (8).

Por ello, el objetivo del presente estudio fue determinar la eficiencia terminal (ET) según modalidad de titulación (MT) de los egresados de pregrado de la Facultad de Estomatología de la Universidad Peruana Cayetano Heredia (FAESTUPCH), Lima-Perú, desde el año 1975 al 2018. 


\section{MATERIAL Y MÉTODOS}

La presente investigación fue retrospectiva basada en los registros de los egresados de pregrado de la FAEST-UPCH desde 1975 hasta el 2018 que se encuentran publicados en el repositorio institucional de la Universidad Peruana Cayetano Heredia (RI$\mathrm{UPCH})$. El periodo de tiempo es determinado desde el primer egresado de la facultad hasta el inicio de la investigación en mayo de 2020, se excluyeron a los egresados 2019 porque sus investigaciones se paralizaron desde marzo hasta diciembre 2020 por la pandemia de COVID-19. Se tuvo un total de 1955 registros de egresados; sin embargo, no se tuvo información de 178 egresados representando una tasa de pérdida de 9,10\%, siendo la muestra final 1777 registros que cumplieron con los criterios necesarios para la investigación.

La MT se categorizó en Tesis (Investigación individual en formato clásico), Trabajo de investigación (TI) (Investigación grupal en formato artículo científico), Servicio Estomatológico Supervisado Alternativo para la Obtención del Título de Cirujano Dentista (SESAOT) (Trabajo de suficiencia profesional basado en servicio comunitario), y Proceso de Suficiencia Profesional (PSP) (Trabajo de suficiencia profesional basado en revisión de la literatura). La ET fue calculada mediante la diferencia de años desde la obtención del título profesional verificado con la publicación del trabajo en el RI-UPCH y el año de egreso, así mismo, se categorizó en "Hasta 1 año" y "De 2 años a más", debe mencionarse que esta fue decisión de los investigadores frente a la ausencia de referencias tomando en consideración que una adecuada ET es la realizada en el menor tiempo posible. Por último, la variable década de egreso se consideró desde el 1975 hasta 2018.

Para construir la base de datos de egresados se accedió a la información disponible en la web oficial de Secretaría Académica de la FAEST-UPCH (https://faest.cayetano.edu.pe/nosotros/organizacion/ secretaria-academica/graduados/promociones). Seguidamente, se procedió a buscar cada uno de los egresados en la web oficial del RI-UPCH (http://repositorio.upch.edu.pe/), este paso permitió completar la base de datos con la información de MT,
ET y década de egreso. Es importante mencionar que este proceso fue posterior a la aprobación del Comité Institucional de Ética de la Universidad Peruana Cayetano Heredia (CIE-UPCH) con fecha 29 de abril del 2020 y código SIDISI N 202105.

Para la construcción de la base de datos se utilizó el programa Microsoft Excel y para el análisis de datos el programa STATA 16.1. El análisis descriptivo permitió obtener las frecuencias absolutas y relativas de las variables cualitativas, y promedios y desviación estándar de las variables cuantitativas. Para el análisis bivariado se usaron las pruebas no paramétricas de Chi-cuadrado, Chi-cuadrado corregida por Yates, U de Mann Whitney y Kruskal Wallis, debido a que los valores cuantitativos no presentaron distribución normal mediante la prueba de Kolmogorov-Smirnov. El estudio contó con un nivel de confianza del 95\%, y un $\mathrm{p}<0,05$.

\section{RESULTADOS}

Se contó con un total de 1777 trabajos de titulación, siendo la Tesis la más frecuente con un $61,17 \%$ $(n=1087)$, seguida de PSP con $18,46 \%(n=328)$, luego el SESAOT con $14,91 \%(\mathrm{n}=265)$ y, por último, TI con $5,46 \%(n=97)$, es importante mencionar que el PSP se cancela en el 2011 y el TI entró en vigor desde los egresados 2017. Según décadas, entre los años 20002009 se evidenció la mayor frecuencia de trabajos de titulación con un 33,31\% $(\mathrm{n}=592)($ tabla 1$)$.

La ET general fue de 1,67 años $(\mathrm{DE}=1,61)$, siendo la más baja de TI con 1,01 años $(\mathrm{DE}=0,10)$ con un máximo de 2 años; seguido del PSP con 1,12 años $(\mathrm{DE}=1,96)$ con un máximo de 26 años; luego Tesis con 1,83 años $(\mathrm{DE}=1,40)$ con un máximo de 14 años, y SESAOT con 1,89 años $(\mathrm{DE}=1,95)$ con un máximo también de 14 años; se encontró diferencia estadísticamente significativa, así mismo, TI presentó diferencia estadística con Tesis y SESAOT $(p<0,05)$. Según décadas de egreso, De 2017-2018 presentó menor ET con un 1,01 años $(\mathrm{DE}=0,10)$ y un máximo de 2 años, y el mayor valor lo presentó De 1980-1989 con 2,58 años $(\mathrm{DE}=3,01)$ y un máximo de 26 años; se encontró diferencia estadísticamente significativa según décadas de egreso, así mismo, De 2017-2018 presentó diferencia estadística con todas las otras décadas $(p<0,05)$ (tabla 2). 
Tabla 1. Modalidad de titulación de los egresados de pregrado de la Facultad de Estomatología de la Universidad Peruana Cayetano Heredia, Lima-Perú, 1975-2018.

\section{Modalidad de Titulación}

Total

n

$\%$

De 1975 a 1979

Tesis

$24 \quad 100,00$

De 1980 a 1989

Tesis

$220 \quad 94,42$

SESAOT (Trabajo de suficiencia profesional-Servicio comunitario)

114,72

PSP (Trabajo de suficiencia profesional-Revisión de la literatura)

20,86

De 1990 a 1999

Tesis

$401 \quad 87,94$

SESAOT (Trabajo de suficiencia profesional-Servicio comunitario)

$51 \quad 11,18$

PSP (Trabajo de suficiencia profesional-Revisión de la literatura)

$4 \quad 0,88$

De 2000 a 2009

Tesis

268

45,27

SESAOT (Trabajo de suficiencia profesional-Servicio comunitario)

$92 \quad 15,54$

PSP (Trabajo de suficiencia profesional-Revisión de la literatura)

De 2010 a 2016

Tesis

SESAOT (Trabajo de suficiencia profesional-Servicio comunitario)

PSP (Trabajo de suficiencia profesional-Revisión de la literatura)

$90 \quad 24,32$

De 2017 a 2018

Trabajo de investigación

$97 \quad 95,10$

SESAOT (Trabajo de suficiencia profesional-Servicio comunitario)

$5 \quad 4,90$

Década de egreso

De 1975 a 1979

$24 \quad 1,35$

De 1980 a 1989

$233 \quad 13,11$

De 1990 a 1999

$456 \quad 25,66$

De 2000 a 2009

$592 \quad 33,31$

De 2010 a 2016

$370 \quad 20,82$

De 2017 a 2018

$102 \quad 5,74$

Modalidad de titulación

Tesis

$1087 \quad 61,17$

Trabajo de investigación

$97 \quad 5,46$

SESAOT (Trabajo de suficiencia profesional-Servicio comunitario)

$265 \quad 14,91$

PSP (Trabajo de suficiencia profesional-Revisión de la literatura)

$328 \quad 18,46$

Total

$1777 \quad 100,00$

n: Frecuencia absoluta.

$\%$ : Frecuencia relativa. 
Tabla 2. Eficiencia terminal en años según modalidad de titulación de los egresados de pregrado de la Facultad de Estomatología de la Universidad Peruana Cayetano Heredia, Lima-Perú, 1975-2018.

\section{Modalidad de Titulación}

Eficiencia Terminal (años)

\section{$\mathbf{X}$}

DE Min Max

De 1975 a 1979

\section{Tesis}

De 1980 a $1989^{*}$

De 1990 a 1999
Tesis
SESAOT (Trabajo de suficiencia profesional-Servicio comunitario)
PSP (Trabajo de suficiencia profesional-Revisión de la literatura)

Tesis

SESAOT (Trabajo de suficiencia profesional-Servicio comunitario)

PSP (Trabajo de suficiencia profesional-Revisión de la literatura)
De 2000 a $2009^{*}$
Tesis

SESAOT (Trabajo de suficiencia profesional-Servicio comunitario)

PSP (Trabajo de suficiencia profesional-Revisión de la literatura)

\section{2,13}

$0,90 \quad 1 \quad 6$

$\begin{array}{cccc}2.07 \mathrm{ab}^{* *} & 1,68 & 0 & 13 \\ 8.82 \mathrm{ac} * * & 3,31 & 3 & 14 \\ 23.50 \mathrm{bc} * * & 3,54 & 21 & 26\end{array}$

$$
1,67
$$

1,48

1,82

1,68

4,00

5,35

$\begin{array}{llll}1.85 \mathrm{ab}^{* *} & 1,26 & 0 & 8 \\ 1.40 \mathrm{ac} * * & 1,03 & 0 & 6 \\ 0.90 \mathrm{bc} * * & 0,65 & 0 & 6\end{array}$

De 2010 a $2016^{*}$

Tesis

SESAOT (Trabajo de suficiencia profesional-Servicio comunitario) PSP (Trabajo de suficiencia profesional-Revisión de la literatura)

De 2017 a 2018

Trabajo de investigación

SESAOT (Trabajo de suficiencia profesional-Servicio comunitario)

$\begin{array}{llll}1.84 \mathrm{a}^{* *} & 1,01 & 0 & 6 \\ 1.68 \mathrm{~b} * * & 0,98 & 0 & 6 \\ 1.07 \mathrm{ab} * * & 0,33 & 0 & 3\end{array}$

Década de egreso*
De 1975 a 1979
De 1980 a 1989
De 1990 a 1999
De 2000 a 2009
De 2010 a 2016
De 2017 a 2018

Modalidad de titulación*

Tesis

Trabajo de investigación

SESAOT (Trabajo de suficiencia profesional-Servicio comunitario)

PSP (Trabajo de suficiencia profesional-Revisión de la literatura)

$\begin{array}{llll}1,01 & 0,10 & 1 & 2 \\ 1,00 & 0,00 & 1 & 1\end{array}$

$\begin{array}{cccc}2.13 \mathrm{abcd}^{* *} & 0,90 & 1 & 6 \\ 2.58 \mathrm{efgh}^{* *} & 3,01 & 0 & 26 \\ 1.70 \mathrm{aeij} * * & 1,58 & 0 & 14 \\ 1.41 \mathrm{bfikl}^{* *} & 1,11 & 0 & 8 \\ 1.61 \mathrm{cgkm}^{* *} & 0,93 & 0 & 6 \\ 1.01 \mathrm{dhjlm} * * & 0,10 & 1 & 2\end{array}$

$\begin{array}{cccc}1.83 \mathrm{ab}^{* *} & 1,40 & 0 & 14 \\ 1.01 \mathrm{ac}^{* *} & 0,10 & 1 & 2 \\ 1.89 \mathrm{~cd}^{* *} & 1,95 & 0 & 14 \\ 1.12 \mathrm{bd}^{* *} & 1,96 & 0 & 26\end{array}$

Total

$1,67 \quad 1,61 \quad 0 \quad 26$

X: Promedio.

DE: Desviación estándar.

Min: Mínimo.

Max: Máximo.

*Prueba de Kruskal Wallis ( $<<0.05$ ).

**Prueba de U de Mann Whitney, letras iguales presentan diferencia estadísticamente significativa $(\mathrm{p}<0.05)$. 
Tabla 3. Eficiencia terminal categorizada según modalidad de titulación de los egresados de pregrado de la Facultad de Estomatología de la Universidad Peruana Cayetano Heredia, Lima-Perú, 1975-2018.

\section{Modalidad de Titulación}

\section{Eficiencia Terminal \\ Categorizada}

Hasta 1 año De 2 años a más

n $\%$ n $\%$

De 1975 a 1979

$\begin{array}{lllll}\text { Tesis } & 2 & 8,33 & 22 & 91,67\end{array}$

De 1980 a $1989 *$

$\begin{array}{lllll}\text { Tesis } & 84 & 38,18 & 136 & 61,82 \\ \text { SESAOT (Trabajo de suficiencia profesional-Servicio comunitario) } & 0 & 0,00 & 11 & 100,00 \\ \text { PSP (Trabajo de suficiencia profesional-Revisión de la literatura) } & 0 & 0,00 & 0 & 0,00\end{array}$

De 1990 a 1999

Tesis

SESAOT (Trabajo de suficiencia profesional-Servicio comunitario)

PSP (Trabajo de suficiencia profesional-Revisión de la literatura)

De 2000 a $2009 * *$

Tesis

SESAOT (Trabajo de suficiencia profesional-Servicio comunitario)

PSP (Trabajo de suficiencia profesional-Revisión de la literatura)

De 2010 a $2016 * *$

Tesis

SESAOT (Trabajo de suficiencia profesional-Servicio comunitario)

PSP (Trabajo de suficiencia profesional-Revisión de la literatura)

De 2017 a 2018

Trabajo de investigación

SESAOT (Trabajo de suficiencia profesional-Servicio comunitario)

Década de egreso**

De 1975 a 1979
De 1980 a 1989
De 1990 a 1999
De 2000 a 2009
De 2010 a 2016
De 2017 a 2018

Modalidad de titulación**

Tesis

Trabajo de investigación

SESAOT (Trabajo de suficiencia profesional-Servicio comunitario)

PSP (Trabajo de suficiencia profesional-Revisión de la literatura)

$\begin{array}{llll}251 & 62,59 & 150 & 37,41 \\ 30 & 58,82 & 21 & 41,18 \\ 2 & 50,00 & 2 & 50,00\end{array}$

$\begin{array}{llll}119 & 44,40 & 149 & 55,60 \\ 63 & 68,48 & 29 & 31,52 \\ 219 & 94,40 & 13 & 5,60\end{array}$

$47,17 \quad 56 \quad 52,83$

$84 \quad 93,33 \quad 6 \quad 6,67$

$96 \quad 98,97 \quad 1 \quad 1,03$

$5 \quad 100,00 \quad 0 \quad 0,00$

$\begin{array}{llll}2 & 8,33 & 22 & 91,67 \\ 84 & 36,05 & 149 & 63,95 \\ 283 & 62,06 & 173 & 37,94 \\ 401 & 67,74 & 191 & 32,26 \\ 203 & 54,86 & 167 & 45,14 \\ 101 & 99,02 & 1 & 0,98\end{array}$

Total

1074

$\begin{array}{llll}525 & 48,30 & 562 & 51,70 \\ 96 & 98,97 & 1 & 1,03 \\ 148 & 55,85 & 117 & 44,15 \\ 305 & 92,99 & 23 & 7,01\end{array}$

n: Frecuencia absoluta.

$\%$ : Frecuencia relativa.

*Prueba de Chi-cuadrado corregida por Yates $(\mathrm{p}<0.05)$.

***Prueba de Chi-cuadrado $(\mathrm{p}<0.05)$. 
La ET “Hasta 1 año" fue de 60,51\% (n=1074) en general, presentando mayores frecuencias TI con $98,97 \%(\mathrm{n}=96)$ y PSP con $92,99 \%(\mathrm{n}=305)$, y $\operatorname{los}$ de menor frecuencia fueron SESAOT con $55,85 \%$ $(n=148)$ y Tesis con $48,30 \%(n=525)$; encontrándose asociación estadísticamente significativa $(\mathrm{p}<0,05)$. Según década de egreso, de 2017-2018 tuvo la mayor frecuencia con $99,02 \%(\mathrm{n}=101)$, seguida de 2000-2009 con $67,74 \%(n=401)$, y en último lugar, el de 1975-1979 con 8,33\% ( $n=2)$; encontrándose asociación estadísticamente significativa $(p<0,05)$ (tabla 3).

\section{DISCUSIÓN}

En el Perú, la denominada antigua Ley Universitaria $\mathrm{N}^{\circ} 23733$ (ALU) otorgaba el GB de forma automática y el TP mediante una Tesis (3), un trabajo de suficiencia profesional (TSP), u otro que considere la institución. Sin embargo, en el año 2014 con la promulgación de la NLU se agregó la necesidad de otra investigación para la obtención del GB (2). Para el caso muy específico de la investigación, todos los registros de egresados se encuentran en el marco de la ALU por lo cual los trabajos de investigación analizados son para la obtención del TP, esto debido a que los primeros egresados en el marco de la NLU son los ingresando 2016 por las diversas modificaciones que ha sufrido la normativa, es decir, para los que egresen recién en el año 2020.

En el año 2017, la Universidad Peruana Cayetano Heredia (UPCH) cambia su modelo de gestión y unifica facultades, originándose administrativa las Facultades de Medicina, de Estomatología y de Enfermería (FMEE-UPCH) con su propia Unidad Integrada de Gestión de Investigación, Ciencia y Tecnología (UIGICT) que agrupa las Unidades de Investigación, Ciencia y Tecnología de cada facultad. Una de las primeras actividades de la UIGICT fue evaluar los procesos de titulación para consensuar una única norma enmarcada en la saliente ALU y la entrante NLU. En el 2017, en la FAEST-UPCH se evidenció una alta eficiencia terminal con muchos egresados que no contaban con título universitario; de un total de 106 egresados de los años 2015 y 2016 , el $61,30 \%(n=65)$ no lo tenían Es así como en esta nueva norma, el SESAOT se mantiene como TSP en la cual es estudiante presenta un informe al finalizar una rotación de servicio comunitario de 4 meses en programas de salud bucal a cargo de FAEST-UPCH; el PSP no es considerado por estar inhabilitado desde 2011 porque realmente no generaba de conocimiento al ser un TSP materializado en una revisión de literatura; y el TI reemplaza la Tesis (9).

Tradicionalmente, la Tesis en la FAEST-UPCH era una investigación original que contemplaba todas las secciones clásicas de un documento como este: introducción, planteamiento de la investigación, marco teórico, objetivos, materiales y métodos, resultados, discusión, conclusiones, recomendaciones, referencias bibliográficas y anexos; se desarrollaba de forma individual bajo la asesoría de un docente y debía ser sustentado ante un jurado. Este proceso demandaba mucho tiempo y muchas veces los alumnos no culminaban la sustentación dejando las investigaciones lo que provocaba que no se titulen, a lo cual se sumaba, los procesos que eran aún más burocráticos con revisiones de documentos que podrían demorar meses (10).

Por ello, la UIGICT propone la modificación a TI, lo cual es aceptado por la FMEE-UPCH en conjunto, lo cual dinamiza la obtención del TP en FAEST-UPCH. Esta modalidad también es una investigación original, sin embargo, el formato es de artículo científico con las secciones de introducción, objetivos, materiales y métodos, resultados, discusión, conclusiones, referencias bibliográficas y anexos; se puede realizar de hasta 3 estudiantes bajo la asesoría de un docente y puede obviarse la sustentación si el trabajo es publicado anteriormente en una revista indexada; a estos cambios se suma que todos los procesos se virtualizan incluyendo la sustentación, un paso previo a este último es la pre-sustentación donde los estudiantes pueden recibir correcciones o desaprobar, pero en la sustentación sólo se califica el tipo de aprobación considerándola una actividad académica formal y pública con un jurado evaluador (11).

Los resultados de la presente investigación confirman que la modalidad de TI ha tenido mejores resultados cerrando la brecha con mayor porcentaje de titulados y reduciendo la ET en comparación con las otras modalidades, siendo importante indicar que al ser un trabajo en formato artículo es más sencillo lograr su publicación, paso final en la difusión del conocimiento que no siempre se obtenía cuando 
se desarrollaban tesis debido a que la reducción del documento era otra etapa que muchas veces el egresado no seguía por priorizar otras actividades profesionales y terminaba siendo responsabilidad del docente, que a veces, tampoco podía culminar. Es así como, las investigaciones son el medio por el cual las universidades generaran su propio conocimiento para entender y cuestionar fenómenos en conjunto con sus estudiantes, donde su crecimiento profesional es fundamental e incrementa las aspiraciones a nuevas metas (11).

Es importante que las autoridades analicen con sumo cuidado la MT con el objeto de lograr una ET adecuada para su universidad. La NLU establece de forma general que es necesario realizar dos investigaciones, una para el GB y otra para el TP; sin embargo, aquellas facultades acreditadas ante el Sistema Nacional de Evaluación, Acreditación y Certificación de la Calidad Educativa (SINEACE) del Ministerio de Educación del Perú, similar a la ALU, pueden determinar modalidades adicionales (2). Por ello, reformulando las directivas, las FMEE-UPCH en el marco de la NLU en 2021, establece que el TI es para la obtención del GB en una modalidad de revisión de literatura y para el TP será la Tesis en versión de investigación original previamente descrito; y la FAEST-UPCH acreditada por SINEACE (13) agrega como modalidades de titulación el TI, la publicación de un artículo original en revista indexada, el SESAOT y el Examen de Suficiencia Profesional (ESP) que evalúa las competencias del egresado, esto debido a las evaluaciones de procesos que se han desarrollado demostrando que realizar una investigación demanda casi un año en culminarse, de agregarse un trabajo adicional se tendría una ET mayor a dos años, lo cual repercute en que los estudiantes no puedan colegiarse, trabajar o seguir estudios de especialización.

Está claro que la ET es una medida crucial de la capacidad de las universidades para insertar en la sociedad a los recursos recientemente formados, este indicador da la pauta para evaluar el desempeño institucional y el proceso formativo (14), a pesar de que no existen referencias respecto a los valores ideales, se plantea hasta un año de ET como criterio adecuado. Sin embargo, como se ha mencionado anteriormente, existen otros factores que pueden determinar esta ET más allá de la MT como los factores económicos, sociales, culturales, y otros
(15), en este estudio no se evaluaron esos factores lo cual es una de las limitaciones, sumado a la pérdida de información.

Finalmente, es importante mencionar que la gestión universitaria debe contemplar la adaptación de las normas a la realidad de los estudiantes, docentes, universidad y el país, la propuesta de una investigación grupal en formato de artículo original es la mejor opción para la obtención de un grado académico o título profesional garantizando una menor eficiencia terminal y contribuyendo al desarrollo científico, permitiendo que los egresados puedan prontamente insertarse laboralmente y ser parte invalorable de la sociedad. Por ello, se concluye en este caso que, el trabajo de investigación presentó mejor eficiencia terminal en los egresados de pregrado de la Facultad de Estomatología de la Universidad Peruana Cayetano Heredia, Lima-Perú, desde el año 1975 al 2018.

\section{Correspondencia:}

Angela Girano-Arévalo

Correo electrónico: angela.girano.a@upch.pe

\section{REFERENCIAS BIBLIOGRÁFICAS}

1. Alvares GM, Figuera GP, Torrado FM. La problemática de la transición bachillerato-universidad en la Universidad de Barcelona. REOP. 2011;22(1):15-27.

2. Congreso de la República. Ley Universitaria Nº30220. Lima: Diario Oficial El Peruano; 2014.

3. Congreso de la República. Ley Universitaria $\mathrm{N}^{\circ}$ N²3733. Lima: Diario Oficial El Peruano; 1983.

4. Martínez MA, Castro SM, Lucena ZM, Zurita OF. Elección de titulación universitaria y expectativas de resultados de los adolescentes de Granada. REOP. 2015;26(3):63-77.

5. Lotkowski VA, Robbins SM, North RJ. The role of academic and non- academic factors in improving college retention. ACT policy report. Iowa: American College Testing ACT Inc; 2004.

6. Gloria AM, Kurpius SER. Influences of self-beliefs, social support and comfort in the university environment on the academic nonpersistence decisions of american indian undergraduates. Cultural Diversity and Ethnic Minority Psychology. 2001; 7(1):88-102.

7. Rodríguez A. Factores que dificultan titularse de una universidad mexicana. Cuad Invest Edu. 2014;5(20):117-27.

8. Zegarra $\mathrm{O}$. Modelo de licenciamiento de los programas de pregrado de Medicina en el Perú. Acta Med Peru. 
2019; 36(4):301-8.

9. Unidad de Investigación, Ciencia y Tecnología/ Facultad de Estomatología/Universidad Peruana Cayetano Heredia. Informe memoria de gestión 20172020. Lima: Facultad de Estomatología, Universidad Peruana Cayetano Heredia; 2020.

10. Unidad de Investigación, Ciencia y Tecnología / Facultad de Estomatología/Universidad Peruana Cayetano Heredia. Informe memoria de gestión Marzo-Agosto 2017. Lima: Facultad de Estomatología, Universidad Peruana Cayetano Heredia; 2017.

11. Unidad Integrada de Gestión de Investigación, Ciencia y Tecnología/ Facultades de Medicina, de Estomatología y de Enfermería. Normas y procedimientos para la elaboración, desarrollo, presentación, evaluación y publicación de trabajos de investigación y tesis. Lima: Facultades de Medicina, de Estomatología y de Enfermería; 2018.
12. Cruz S, Sandi JC. Importancia de la educación superior en el desarrollo profesional para la población estudiantil. La Plata: Universidad Nacional de La Plata; 2012.

13. Sistema Nacional de Evaluación, Acreditación y Certificación de la Calidad Educativa. Resolución de Presidencia $\quad \mathrm{N}^{\circ} 000295-2020$-SINEACE/CDAH-P. Lima: Ministerio de Educación; 2020.

14. Pérez ML. Impacto de la eficiencia terminal de la educación superior en México en la economía educativa. Eseconomía. 2016;11(45):133-56.

15. Cuéllar O, Bolívar AG. ¿Cómo estimar la eficiencia terminal en la educación superior? Notas sobre su estatuto teórico. Rev Educ Sup. 2006;35(139):7-27.

Recibido : 15-01-2021

Aceptado : 11-06-2021 